\title{
Fach- und Führungskräfte halten und gewinnen
}

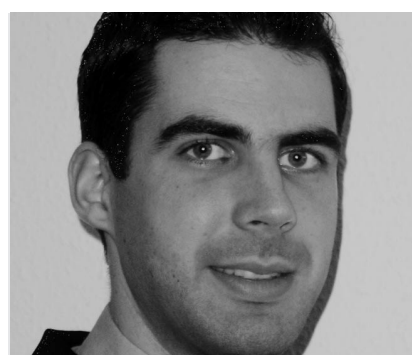

VON THOMAS MÜLLER

Müller ist als Kompetenzkoordinator zuständig für die Personalberatung der contec $\mathrm{GmbH}$. Er arbeitet seit 2006 bei der Beratungsfirma und unterstützt Unternehmen der Gesundheits- und Sozialwirtschaft bei der Abdeckung von Vakanzen, bei der Gewinnung von Fach- und Führungskräften, im Personalmarketing und in der gesamten Personalprozesskette. Müller ist zudem kooptiertes Vorstandsmitglied der Akkreditierungsagentur für Studiengänge im Bereich Gesundheit und Soziales AHPGS e. V.

E-Mailmueller@contec.de
Viele soziale Organisationen finden angesichts der demografischen Entwicklung inzwischen nur schwer qualifiziertes Personal. Ein systematisches und kontinuierliches Personalmarketing wird für manche Sozialunternehmen zur Überlebensfrage.

Künftig wird es in der Sozialwirtschaft eine Unterscheidung geben zwischen denjenigen Unternehmen, die im Hinblick auf ihr Personal zukunftsträchtig und zielführend aufgestellt sind und denjenigen, deren personalwirtschaftliche Risiken die Existenz gefährden.

Strategisch werden die Mitarbeitergewinnung, dauerhafte Bindung und erfolgreiche und langfristige Personalentwicklung in Zeiten des demografischen Wandels (vgl. Raffelhüschen 2008) und des Fach- und Führungskräftemangels zum entscheidenden Wettbewerbsfaktor. Organisationen der Sozialwirtschaft müssen bei ihrer wichtigsten Ressource zum einen in ihr Personalmarketing und in nichtmonetäre Personalbindungs- und Personalentwicklungsinstrumente investieren, um dauerhaft ihren Personalbedarf adäquat decken zu können. Zum anderen müssen sie gegen die oftmals attraktivere Bezahlung in der Privatwirtschaft kämpfen. Bereits heute haben Entscheider in der Sozialwirtschaft erhebliche Probleme, vakante Stellen qualifiziert und adäquat nach zu besetzen.

Doch der Status quo der Personalakquisition lässt derzeit oftmals erkennen: Personalmarketing, aber auch Personalführung werden noch »allzu sehr vernachlässigt. Gerade bei Einrichtungen mittlerer Größenordnung wird kaum Personalkapazität für diese Aufgabe vorgehalten. Grundsätzlich wird der Leiter der Einrichtung (...) als zuständig erklärt. Untersuchungen besagen jedoch, dass die Führungskräfte von Alltagsproblemen serschlagen ' werden und nur noch >Feuerwehrfunktionen` ausüben, so dass kaum
Zeit für eine strategische Personalentwicklung (...) -förderung (... und -marketing; Zusatz d. Verf.) verbleibt. (...) Dies muss sich - auch angesichts stetig weiter wachsender Anforderungen bei sich verschlechternden Stellenschlüssel und einer drohenden Knappheit an qualifizierten Kräften in der Zukunft - umgehend verändern, wenn nicht eine gravierende Einschränkung der Qualität hingenommen werden soll. « (Becker 2002, 94; Auslassungen durch den Verfasser)

Personalarbeit und das Gewinnen qualifizierter Fach- und Führungskräfte in der Sozialwirtschaft wird oft als Verwaltungsund nicht als Gestaltungsaufgabe verstanden. Die Situation auf dem immer stärker werdenden Nachfragemarkt »Personal « zeigt, dass es weniger um die Entscheidung eines Unternehmens für oder gegen einen Bewerber geht. Vielmehr liegt diese Entscheidung längst beim Bewerber: Dieser entscheidet sich für oder gegen ein Unternehmen (vgl. Müller-Benz 2008, 28). Die Analyse und Bearbeitung von Themenfeldern wie Employer Branding, Employee Branding und Employer-Value-Proposition (vgl. Kasten Seite 34 oben) wird in der Sozialwirtschaft zum entscheidenden Wettbewerbsfaktor.

Betrachtet man nämlich die Mitarbeitenden in der Sozialwirtschaft, so lässt sich konstatieren: "Mehr als in anderen wirtschaftlichen Bereichen ist (...) (... hier, Zusatz des Verfassers) eine enge Verknüpfung von Profession und Persönlichkeit gegeben. Mehr als anderswo wird von professionellen Helfern erwartet, dass sie mit ihrer Persönlichkeit das ausfüllen, was die Profession an Handlungskompetenz verspricht. Bezogen auf die professionelle Ausrichtung müssen sozialarbeiterische (...) Fachkompetenzen mit betriebswirtschaftlichen Kompetenzen verknüpft und zu einem integrierten Handlungskonzept verbunden werden. « (Mantel 2002, 212; Auslassungen durch den Verfasser)

Wie können nun Organisationen der Sozialwirtschaft diesen Spezifika der Mit- 
arbeitenden Rechnung tragen und die für sie passenden Führungskräfte gewinnen

Dafür ist ein systematisches und kontinotwendig, das die spezifischen Belange der jeweiligen Organisation berücksichtigt. Hier wird Personalmarketing verstanden als » Orientierung der gesamten Personalpolitik eines Unternehmens an den Bedürfnissen von gegenwärtigen und zukünftigen Mitarbeitern mit dem Ziel, gegenwärtige Mitarbeiter zu halten, zu motivieren und neue Mitarbeiter zu gewinnen « (Simon et al. 1995, 13 ff.). Primäre Intention des Personalmarketings als Teil des gesamten (Personal-) Management-Systems ist es mithin, Organisationen mit qualifizierten und motivierten Mitarbeitenden zu versorgen (vgl. Süß 1996, 10 ff.). Differenziert wird zwischen internem und externem Personalmarketing.

\section{Bindung vorhandener Mitarbeitender}

Aktivitäten des internen Personalmarketings beziehen sich auf bereits vorhandenes Personal. Im Mittelpunkt steht beispielsweise das Image einer Organisation, welches durch das Leitbild getragen wird. Instrumente der Personalentwicklung, der Fort- und Weiterbildung (z. B. mit »Förderpools «) und das Aufzeigen individueller Karrierepfade (d. h. Aufstiegsmöglichkeiten in einer Fach- oder Führungskarriere) sind hierbei Erfolgsfaktoren. Gelingen kann eine zielführende und zukunftsträchtige Personalentwicklung durch die Konzeption sogenannter Personal-Portfolios. In diesen werden Leistung und Qualifikation des Personals kontinuierlich bewertet (vgl. Abb. 1). Vorgesetzte erhalten so Auskunft über die Qualität und Ausgewogenheit der Mitarbeitenden im Unternehmen sowie Entscheidungshilfen hinsichtlich erforderlicher Beschaffungs-, Anreiz- und Entwicklungsstrategien.

Neben monetären Leistungsanreizen (Grundgehalt, Boni, erfolgs- und zielorientierter Anteil etc.) und Sozialleistungen und langfristig binden? nuierliches Personalmarketing-System

(z. B. zusätzliche Altersfürsorge, vermögenswirksame Leistungen etc.) spielen insbesondere nichtmonetäre Leistungen einer Organisation eine entscheidende Rolle bei der Bewertung der Attraktivität eines Arbeitgebers. Subsumiert werden hierbei in erster Linie eine Motivation stiftende Unternehmenskultur, in der Fairness und Respekt essentielle Bestandteile sind.

Mitwirkungsmöglichkeiten der Mitarbeitenden (z. B. Umgang mit Verbesserungsvorschlägen, Partizipations-, Verantwortungs- und Autonomiegrade etc.), Vertretungsregelungen, Informationsbereitstellung, Umgang mit Konflikten und Fehlern sowie spezielle Sozialleistungen (z. B. Kinderbetreuung, flexible Arbeitszeitmodelle, Dienstfahrzeug, Handy- oder Laptop, Sabbatical-Möglichkeiten etc.) gewährleisten eine erfolgreiche Bindung vorhandenen Personals.

Elemente der Job Rotation (Arbeitsund Aufgabenwechsel), des Job-Enrichments (Aufgabenanreicherung zur Höherqualifikation der Mitarbeitenden) sowie Job Enlargement (Aufgabenerweiterung um strukturell gleichartige Aufgaben) runden das interne Personalmarketing ab.

\section{Gewinnung qualifizierter Fach- und Führungskräfte}

Das externe Personalmarketing verzahnt sich mit den Elementen des internen Personalmarketings. Externes Personalmarketing bezieht sich auf das neu zu gewinnende Personal. Infolgedessen liegt der Fokus auf der Attraktivität einer Organisation auf dem (externen) Arbeitsmarkt. Da aber systemimmanente Stärken beziehungsweise eine attraktive Unternehmenskultur potenziellen Bewerbern oftmals nicht bekannt sind, müssen diese durch entsprechende Maßnahmen von der Attraktivität der Organisation überzeugt werden.

Doch was können soziale Unternehmen konkret tun, um eben diese Führungskräfte für sich zu gewinnen? Zunächst sollten soziale Organisationen den Status quo ihrer Arbeitgeberattraktivität und ihres Personalmarketings bestimmen. Diese Si-

\begin{tabular}{|c|c|c|}
\hline Hohe Leistung & $\begin{array}{l}\text { "Arbeitstiere«: } \\
\text { Mitarbeitende, die stark an das } \\
\text { Unternehmen gebunden sind, } \\
\text { aber nur über ein geringes } \\
\text { Entwicklungspotential verfügen }\end{array}$ & $\begin{array}{l}\text { "Stars«: } \\
\text { hochmotivierte Mitarbeitende, die } \\
\text { dem Unternehmen ihr } \\
\text { Fähigkeitspotential längerfristig zur } \\
\text { Verfügung stellen. }\end{array}$ \\
\hline Geringe Leistung & $\begin{array}{l}\text { » Leistungsschwache«: } \\
\text { Mitarbeitende, die eine tiefe } \\
\text { Entwicklungsmotivation } \\
\text { aufweisen. }\end{array}$ & $\begin{array}{l}\text { »Problemfälle«: } \\
\text { Mitarbeitende, von denen man nicht } \\
\text { weiß, ob sie längerfristig im Betrieb } \\
\text { bleiben möchten. }\end{array}$ \\
\hline & Geringes Potenzial & Hohes Potenzial \\
\hline
\end{tabular}

Abb. 1: Aufbau eines Personal-Portfolios

Quelle: eigene Darstellung, vgl. Bühner 1997, 132; vgl. auch Berthel 2000, 221 ff.

\section{Drei Fragen, denen sich personalsuchende Arbeitgeber stellen müssen}

- Employer Branding: Wie wird die Organisation als Arbeitgeber auf dem Arbeitsmarkt wahrgenommen?

- Employee Branding: Wie prägen Meinungsäußerungen der Mitarbeitenden das Image und die Marke eines Arbeitgebers?

- Employer-Value-Proposition: Wodurch unterscheidet sich die Organisation von anderen Arbeitgebern? Was macht sie einzigartig und attraktiv für Mitarbeitende?

tuationsanalyse kann damit beginnen, statistische Angaben zur seitherigen Personalrekrutierung bei vakanten Stellen zu erfassen, zu analysieren und zu bewerten, beispielsweise:
- Anzahl der Bewerbungen
- Qualität der Bewerbungen
- Dauer des Besetzungsprozesses
- Fluktuation in der Probezeit
- Mitarbeiterzufriedenheit
- Fehlzeiten

Diese Daten als exemplarische Indikatoren zur Bewertung der Arbeitgeberattraktivität sollten mit externen Benchmarks in Relation gesetzt werden. Als mögliche Quelle dieser Benchmarks können Veröffentlichungen des jeweiligen Spitzenverbandes, der Bundesagentur für Arbeit, der Statistik-Behörden, branchenspezifischer Personalberatungen oder Daten von früheren Personalakquisitionen dienen. Des weiteren empfiehlt sich der kontinuierliche Austausch mit anderen Einrichtungen und daran anknüpfend eine strategische Allianz mit diesen.

$\mathrm{Zu}$ Beginn eines erfolgreichen Personalmarketings steht mithin neben der Bestimmung von Zieldimensionen, der Festlegung von Meilensteinen, des prozessorientierten Zielcontrollings und der Benennung von Verantwortlichkeiten, die Definition von Zielgruppen. Je nachdem ob eine Organisation beispielsweise Studenten (national oder international), Hochschulabsolventen (national oder 
Grafisch ansprechende Stellenanzeigen

Absolventenkongresse

Traineeprogramme

Austrittinterviews

Leadershipprogramme

Kontaktpflege zu Aus- und Weiterbildungsinstitutionen

Ansprechender, barrierefreier und interessanter

Internetauftritt mit leicht

findbaren Stellenanzeigen

Aufbau und Pflege eines qualifizierten Bewerberpools

Mentoring-, Coaching- und Einarbeitungs- Programme

Berufsbegleitendes Studium

$$
\text { e-Recruting }
$$

Kontaktpflege bei Schulen

Teilnahme an

Schulinformationstagen

Gezielte Flyer

Förderung von

Initiativbewerbungen
Demografieorientierte Arbeitsplätze

Recruiting-Workshops

Praktikantenprogramme

Doktorandenprogramme

Fachmessen - und Kongresse

$$
\text { Masterprogramme }
$$

Attraktive und

aussagekräftige

Informationsbroschüren

Veröffentlichung von

Fachpublikationen und

Praxisberichten

Teilnahme an Wettbewerben (z.B. great place to work)

Partnerschaften zu

Einrichtungen im In- und Ausland

Berufsbegleitende Ausbildung

(elektronische) Jobportale

Talentmarketing

Nutzung von

Branchennetzwerken

Talent Relationship Management
Diplomantenprogramme

Stipendien

Imageanzeigen

Mitarbeitendengespräche

Kontaktpflege zu

Zielhochschulen

Teilnahme an

Zertifizierungen (z.B. Audit

Beruf \& Familie,

Qualitätsmanagementsystem)

Innovative Gehaltsmodelle (z.B. Cafeteria-Systeme)

Ausschreibung von Forschungsarbeiten

Ideenmanagement

Firmenkontaktmessen

Individuelle Karrierepfade

Zielgruppenorientierte Mailings (z.B. an AlumniNetzwerke)

Gesundheitsmanagement
$[\ldots]$
$[\ldots]$

Abb. 2: Exemplarische Instrumente des Personalmarketings

international), Young Professionelles (national oder international), Fach- und Führungskräfte mit Berufserfahrung (national oder international), alle aktuell in der Organisation beschäftigten Mitarbeitende oder ausgewählte aktuell beschäftigte Mitarbeitende in den Fokus ihrer Personalmarketingaktivitäten stellt (vgl. Deutsche Gesellschaft für Personalführung e.V. 2004, 16 ff.), muss die Personalstrategie different ausgerichtet sein. Dabei können zahlreiche Instrumente erfolgsversprechend zum Einsatz kommen, um seinen Personalbedarf passgenau decken zu können (vgl. Abb. 2).

$\mathrm{Da}$ indes viele soziale Organisationen (aufgrund ihrer Größe) weder die zeitlichen noch finanziellen Ressourcen haben, ein systematisches und umfassendes Personalmarketing und beispielsweise einen qualifizierten Bewerberpool zu pflegen, sind strategische Allianzen mit anderen Einrichtungen oder der Rückgriff auf unabhängige professionelle Personaldienstleister für die dauerhafte Erfolgssicherung ein möglicher Schritt.
$\mathrm{Zu}$ beachten ist ferner: Nicht alle genannten Instrumente des Personalmarketings sind für jede Organisation zielführend. Für einige kann strategischer Mehrwert auf dem Arbeitsmarkt bereits dadurch geschaffen werden, dass Kontakt zu ehemaligen Mitarbeitenden (Praktikanten, Auszubildende, Mitarbeitende) gehalten wird. Dies kann durch das regelmäßige Versenden der Hauspublikation, das Einladen auf die Weihnachtsfeier oder durch eine Glückwunschkarte zum Geburtstag geschehen. Bei anderen ist ein abgestimmter und wohlüberlegter Einsatz mehrerer Instrumente erforderlich. In jedem Fall sollten diese Instrumente selektiert, priorisiert und integraler Bestandteil der Personal- und infolgedessen der Gesamtunternehmensstrategie werden.

Die Personalmarketingstrategie muss als Teil der Unternehmensstrategie im strategischen Managementprozess systematisch erarbeitet werden. Die Bildung einer attraktiven Arbeitgebermarke (mit Wiedererkennungseffekten) setzt eine stringente Kommunikationspolitik (Public Re- lations) und eine Einbettung in die Corporate Identity voraus.

Innerorganisatorisch sollte der Informationsfluss zwischen Top-Management, der Mitarbeitervertretung und den Mitarbeitenden sicher gestellt und ein systematisches Controlling-System zur Steuerung der Maßnahmen implementiert sein.

\section{Fazit}

Die Gewinnung qualifizierter Führungskräfte wird zukünftig an die Güte des Personalmarketingsystems gekoppelt sein und setzt ein aktives Werben sozialer Organisation um Führungskräfte voraus. Wesentlich zum langfristigen Erfolg ist die Entwicklung einer werteorientierten Unternehmenskultur, in der Glaubwürdigkeit, Fairness und Respekt, sowie die Gesundheit am Arbeitsplatz eine tragende Rolle spielen. Bereits heute gilt es für soziale Organisationen daher in diesen Bereich zu investieren, um langfristig am Markt bestehen zu können.

\section{Literatur:}

Becker; H. E. (2002): Das Sozialwirtschaftliche Sechseck - Grundzüge. In: Becker, H. E. (Hg.) (2002): Das Sozialwirtschaftliche Sechseck. Freiburg, 17-108.

Berthel, J. (2000): Personal-Management. Grundzüge für Konzeptionen betrieblicher Personalarbeit. Stuttgart.

Bühner, R. (1997): Personalmanagement. Landsberg/Lech.

Deutsche Gesellschaft für Personalführung

e. V. (Hg.) (2004): Personalmarketing - ein unterschätzter Erfolgsfaktor. Ergebnisse einer Tendenzbefragung. Praxis Papiere Ausgabe 8/2004. Düsseldorf.

Koziol, K.; Pförtsch, W.; Heil, S.; Albrecht, K. (2006): Social Marketing. Erfolgreiche Marketingkonzepte für Non-Profit-Organisationen. Stuttgart.

Mantel, H. (2002): Die Mitarbeiterorientierung. In: Becker, H. E. (Hg.) (2002): Das Sozialwirtschaftliche Sechseck. Freiburg, 211-241. Müller-Benz (2008): Unternehmen werben um gutes Personal. In: Neue Caritas, Heft 13/2008. Freiburg im Breisgau, 28.

Raffelshüschen, B. (2008): Pflege 2030 Einflussfaktoren auf die demografische Entwicklung in der Pflege. Vortrag am 5. Juni 2008 auf dem Deutschen Pflegekongress 2008 im Rahmen des Hauptstadtkongresses 2008 Medizin und Gesundheit vom 4. bis 6. Juni 2008 in Berlin.

Simon, H.; Wiltinger, K.; Sebastian, K.-H. (1995): Effektives Personalmarketing. Strategien - Instrumente - Fallstudien. Wiesbaden. Süß, M. (1996): Externes Personalmarketing für Unternehmen mit geringer Branchenattraktivität. München und Mering. 